\title{
Verifying Software Requirements Characteristics Based on Rules Defined from Software Component Relationships
}

\author{
Nattapon Phanthanithilerd and Nakornthip Prompoon
}

\begin{abstract}
In identifying software requirements from users, the requirements are usually in the form of natural language sentences, which may be ambiguous. Therefore, a language has been developed to model the software in different aspects help solve such problem and communicate between the users and developers, namely, Unified Modeling Language (UML). Using UML for Software designing from natural language sentences may be complicated, and the software developers may not identify the requirements consistently; therefore, software developers should give importance to verifying that the requirements in the natural language sentence format and in the model format have good properties. Therefore, this research proposes rules for verifying that requirements in the natural language sentence format and requirements in the model have good properties according to the IEEE 830 standard, namely, unambiguity, consistency and traceability, Applying the proposed rules is beneficial to ensure that the requirements identified in the model are consistent with the user requirements in the natural language sentence format, allowing the software developers to develop the software according to the user requirements.
\end{abstract}

Index Terms-Software requirement properties, software model, requirements verification.

\section{INTRODUCTION}

Identifying user requirement is important for specifying the scope of software development. The content of the requirements identification is often in the form of natural language sentences, which specify the overview of the function, such as function for system access, function for membership sign up, etc., as well as identifying data that the system must store, such as user attributes and user usage data etc. However, natural language sentences are often ambiguous. One sentence may be interpreted more than one meaning. Therefore, using a notion like UML for software modeling help resolve such issue and software design artifacts may be used for communication between users and developers.

UML can be used for creating a software model in different aspects during the requirements engineering process, by taking the natural language sentences identifying user requirements and specifying them in the model. To model the software in order to illustrate the function model and structural model, use case diagrams and class diagrams are often used respectively.

Manuscript received August 25, 2014; revised November 4, 2014.

Nattapon Phanthanithilerd and Nakornthip Prompoon are with the Department of Computer Engineering, Faculty of Engineering, Chulalongkorn University, Bangkok, Thailand (e-mail: nattapon.pha@student.chula.ac.th).
Use case diagrams show the capability of the system for serving the user or other systems, and the class diagram shows the details of the data stored in the system. To ensure that the use case diagrams and the class diagrams have detailed and clear data, use case descriptions and class responsibilities and collaborators cards (CRC cards) are created, showing the various details in both respective diagrams.

However, designing a model from natural language sentences may be complicated, as software developers may not identify both types of requirements consistently, and the property of natural language sentences often be ambiguous may create inconsistency. Therefore, software developers have to give importance to verifying the two types of requirements to be consistent, requiring each requirement to be traceable, as well as verifying for ambiguity that may occur in natural language sentences, by using good properties specified in the IEEE 830 standard [1] to specify the verification context.

The IEEE 830 standard, Recommended Practice for Software Requirements Specification, specifies 8 good properties of requirement specifications: Correctness, Unambiguity, Completeness, Consistency, Ranking for importance and stability, Verifiability, Modifiability, and Traceability. This research focuses on verifying 3 properties: 1) Unambiguity, as a property of natural language sentences is ambiguity, if a natural language sentence has the Unambiguity property, it will allow the software developers to accurately specify the model to the user requirements. 2) Consistency, as when designing a model from natural language sentences, which may be complicated, the software developer should therefore verify both lists of requirements to reduce conflict between requirements 3 ) Traceability, because some systems may have many user requirements, which may result in incomplete specification of those requirements. Therefore, tracing will allow software developers to know whether the requirements specified in the model are complete, or which parts are missing or added.

Therefore, this research proposes rules for verifying requirements in the natural language sentence format and requirements in the model format to have good properties according to the IEEE 830 standards: unambiguity, consistency and traceability. The proposed rules are created from the relationships of the various components of the natural language sentences and the model, according to the following relationships: 1) The relationship between the natural language sentences and the model, namely, the relationship between the functional natural language sentences and the use case model, and the relationship between the structural natural language sentences and the class model. 2) The relationships within the model, that is, the 
relationships between the use case model and the use case description, and the relationships between the class model and the class description. 3) The relationship between the functional model and structural model, that is, the relationship between the class model and the use case model.

The rest of the paper is organized as follows: Section II and Section III will discuss related research and theories. Section IV will explain the research method, and the last section, Section V, summarizes the research and future research approaches.

\section{RELATED RESEARCH}

Various works related to requirements verification have been studied as follows:

The research in [2]-[4] proposes concepts for verifying the consistency of logical or temporal contradictions, using a formal method. The research in [2] verifies consistency between the use case model and the class model. The research in [3] verifies consistency between the class model and the state machine diagram, and the research in [4] verifies consistency between the class model and the Prolog script. However, these works of research have limitations due to their formal methods, which cannot be applied effectively to natural language sentences for verification with the model. Therefore, the researchers have done research on methods for verifying natural language sentences with the software model defined by using UML.

The research in [5], [6] proposes methods for applying the Semantics of Business Vocabulary and Business Rules (SBVR) to create relationships with natural language sentences. The research in [5] aims to reduce the ambiguity of natural language sentences, and the research in [6] aims to apply relationships for use in creating class models. The results from creating relationships between natural language sentences and SBVR produce an average F-Value of higher than $80 \%$. Therefore, the researchers apply this method as a basis in creating relationships between natural language sentences and models for more effectiveness in verification.

From studying works related to this research, this research applies the principles for verification of 3 properties, these are, unambiguity, consistency, and traceability, by creating rules from the relationships of the components of each requirement for verification of the 3 properties.

\section{UNDERLYING CONCEPTS}

\section{A. Software Models}

This research focuses on functional models, which are presented in the form of use case diagrams and structural models, presented as class diagrams. In order to describe the details of use case models and class models, use case descriptions and class descriptions are created and used for verification. The rules used for verification take each component of the diagram and description and create a relationship with natural language sentences, because in the requirements engineering process, both models are commonly used to identify user requirements. The components of the models and the descriptions used for verification are as follows:

\section{1) Use case model}

The use case model is used to show the overall behavior and services provided by the system. The components used for verification are shown in Table I.

TABLE I: COMPONENTS IN THE USE CASE MODEL [7]

\begin{tabular}{|l|l|}
\hline Name & Symbol \\
\hline A use case & \\
\hline An actor & \\
\hline A subject boundary & \\
\hline An association relationship & $\checkmark<<$ <includes> \\
\hline An extend relationship & $\checkmark<$ <extends>> \\
\hline An generalization relationship & \\
\hline
\end{tabular}

\section{2) Class model}

The class model is used to show the data, as well as the properties of the data stored by the system. The components used for verification are shown in Table II.

TABLE II: COMPONENTS IN CLASS MODEL [7]

\begin{tabular}{|l|l|}
\hline Name & Symbol \\
\hline A class & $\begin{array}{c}|c| \\
\frac{\text { Class1 }}{\text {-Atribute-1 }} \\
\text { +Operation-10) }\end{array}$ \\
\hline An attribute & $\begin{array}{l}\text { Attribute name /derived attribute } \\
\text { name }\end{array}$ \\
\hline A operation & Operation name() \\
\hline An association relationship & \\
\hline An aggregation relationship & \\
\hline An composition relationship & \\
\hline An generalization relationship & \\
\hline
\end{tabular}

TABLE III: COMPONENTS IN USE CASE DESCRIPTION [7]

\begin{tabular}{|l|l|}
\hline Name & Details \\
\hline Name & Name of use case \\
\hline Actor & Caller for use case \\
\hline Description & Use case description \\
\hline Relation & $\begin{array}{l}\text { Relationship between use case and } \\
\text { other use cases }\end{array}$ \\
\hline Normal flow & Use case procedure \\
\hline Sub flow & Use case sub-procedure \\
\hline Alternative flow & $\begin{array}{l}\text { Alternate procedures to the normal } \\
\text { flow of the use case }\end{array}$ \\
\hline
\end{tabular}

\section{3) Use case description}

In order to make the use case model clear and show the details of the model, a use case description is used. A use case description has a 1-1 relationship with the use case diagram components. The description has components as shown in Table III. 


\section{4) Class description}

To make the class model clear and display the details of the model, a class description is used. A class description has a 1-1 relationship with the components of the class in a class diagram. The class description has components as shown in Table IV.

TABLE IV: COMPONENTS IN CLASS DESCRIPTION [7]

\begin{tabular}{|l|l|l|}
\hline Part & Name & Details \\
\hline \multirow{5}{*}{ Front } & Class name & Name of the class \\
\cline { 2 - 3 } & Description & Details of the class \\
\cline { 2 - 3 } & Responsibilities & Duties of the class \\
\hline \multirow{3}{*}{ Back } & Attribute & $\begin{array}{l}\text { Classes related to the class in order } \\
\text { to perform a specific } \\
\text { function/service }\end{array}$ \\
\cline { 2 - 4 } & Relation & $\begin{array}{l}\text { Internal attributes of the class. } \\
\text { Compose of attribute name, type } \\
\text { and scope of attribute }\end{array}$ \\
\hline & $\begin{array}{l}\text { Relationships to other classes, } \\
\text { consisting of 3 relationships: } \\
\text { association relations, aggregation } \\
\text { relations, and generalization } \\
\text { relations. }\end{array}$ \\
\hline
\end{tabular}

\section{B. Good Properties of Requirements Specifications}

Good properties of requirements specifications are significantly concerned for creating requirements specifications with quality. Eight good properties of requirements specifications have been defined in the IEEE 830 standard (Recommended Practice for Software Requirements Specification) [1], of which this research gives importance to only unambiguity, consistency and traceability, as shown in Table V.

\begin{tabular}{|c|c|}
\hline Property & Details \\
\hline Unambiguity & $\begin{array}{l}\text { Each requirement shall only be interpreted with } \\
\text { one meaning }\end{array}$ \\
\hline Consistency & $\begin{array}{l}\text { Requirements are consistent within the document. } \\
\text { The requirements specification document has } \\
\text { consistency if there are no contradictions as } \\
\text { follows: } \\
\text { 1. Contradictions in attributes specified in the } \\
\text { real world } \\
\text { 2. Logical and temporal contradictions for } \\
\text { actions in the system } \\
\text { 3. Contradictions in using different words to } \\
\text { describe the meaning of the same object }\end{array}$ \\
\hline Traceability & $\begin{array}{l}\text { Each requirement shall have an origin and be } \\
\text { traceable. Tracing is categorized into two types: } \\
\text { 1. Forward tracing } \\
\text { 2. Back tracing }\end{array}$ \\
\hline
\end{tabular}

\section{RESEARCH METHOD}

The research method is separated into 6 steps: 1) Study and specify the context for properties used for verification 2) Study natural language sentence structure 3) Study the components of each UML model, including the model description 4) Create the relationship for each requirement from the components that have been studied 5) Create rules according to the context of properties from the components of each requirement 6) Evaluate the rules used for verification, as shown in the overview of the research in Fig. 1.

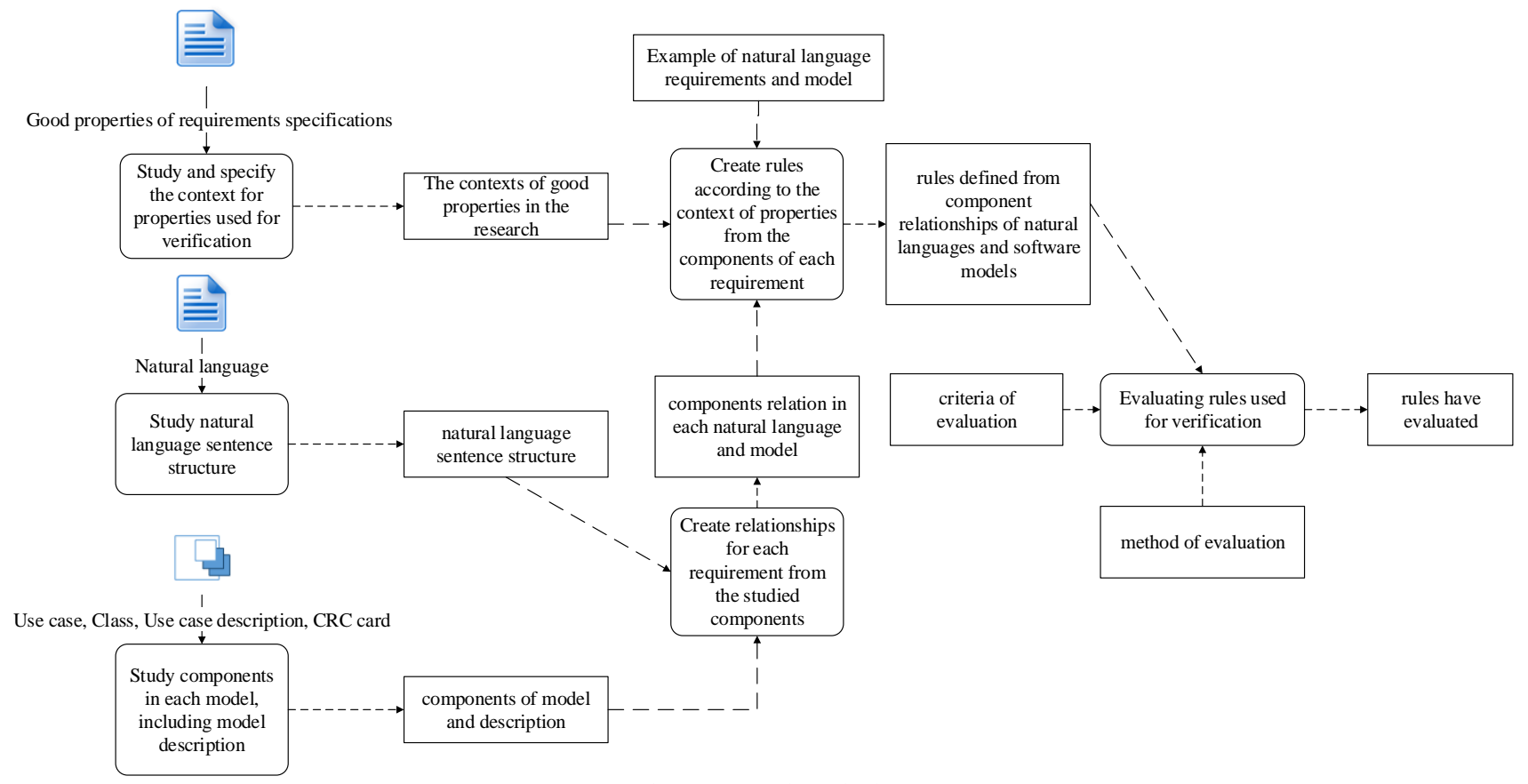

Fig. 1. Research method.

\section{A. Study and Specify the Context for Properties Used for Verification}

This step involves studying good properties according to the research in [1], which focused on the unambiguity, consistency, and traceability properties. The context of the research is then specified to make the rules for verification. The contexts of good properties in the research are as follows:

\section{1) Unambiguity}

Namely, the requirements in the natural language sentence format shall not have immeasurable qualifiers, such as 
"Library system will respond to user quickly". The sentence has the qualifier quickly, which cannot be measured as time, resulting in ambiguity.

\section{2) Consistency}

Namely, the requirement is only consistent when the list of requirements does not have contradictions in the context of the research. In this research, three types of contradiction have been defined, as follows:

1) Contradictions in relationships in tokens between requirements in natural language sentence format and components with the model, focusing on verification relating to the relationship between two or more tokens in each requirement. For example, a natural language sentence represents requirements as: "The user can borrow two kinds of loan items, book and CD", while in the class diagram, there exists the book class with the inheritance relationship with the loan items class, but there does not exist a CD class with the inheritance relationship with the loan items class, etc.

2) Contradictions in order of calling during the service of the system, and contradictions of natural language sentences with the multiplicity value in the class diagram are the focus on verification of the order in calling functions and multiplicity specifications. For example, a natural language sentence states the specification for borrowing books: "It is possible that each user may borrow at most 8 items", while the class diagram indicates a relationship between the user and loan items classes, but not a multiplicity value of $1-0.8$, etc.

3) Contradiction of words and role of tokens in natural language sentences and model components are the focus on verifying words and their roles between the requirements in various forms. For example, the natural language sentence shows a noun indicating a property of a book, "author", while the class diagram does not show an attribute named "author" in the book class etc.

\section{3) Traceability}

Namely, requirements must be able to be referenced, and traced forward (natural language sentence to model); for example, a natural language sentence exists that references borrow, an action done to a book, so in the use case diagram, there must be a "borrow book" use case etc.; as well as can be traced backwards (model to natural language sentence); for example, there exists a borrow book use case, so there must exist a natural language sentence that references borrow, an action done to a book etc.

The result of this step produces good properties to be used for verification according to the context of the research. The created rules shall have a verification scope according to the specified context of the research.

\section{B. Study Natural Language Sentence Structure}

This step involves the study of the structure of natural language sentences for use in creating relationships with the model components. The natural language sentences of interest are functional and structural natural language sentences. The research in [8]-[10] introduces a format for writing good functional natural language sentences, that is: "The $<$ operator role $>$ shall be enabled to $<$ do the required action $>$."The operator role" is the role that can use the function and "do the required action" is the service provided by the system. We will split the format into components for use in creating relationships with the functional model.

In addition, the software developer should write structural natural language sentences to clearly identify the information that should be stored by the system, as well as the properties of the data used for storage. However, the structural natural language sentence does not have a fixed form for writing. Thus, we will take the components of the structural natural language sentence and create relations with the structural model.

\section{Study Components in Each Model, Including Model Description}

This step involves the study of the components in the models, namely, the use case model and the class model, including the descriptions from both models. The components obtained from the study will be used to create the relationship with the structures of the natural language sentence, as well as the relationship between the structural model and the functional model, and the model and the description. The components of the model and the descriptions consist of components as mentioned in section 3.1, underlying concept.

\section{Create Relationships for Each Requirement from the Studied Components}

This step involves taking the studied components, consisting of the components of the natural language sentence, and the components of the description, to create 3 relationships as follows: 1) Relationship between natural language sentences and the model, for example, "Customer should be able to create new borrow order": "Customer" is a noun acting as the subject of the sentence, and will be created into an Actor, and "create new borrow order" is a verb related with the subject, and will be created into a Use case in the Use case diagram. "A book has title": "Book" is a noun in the sentence, and will be created into a class, and "title" is a noun related with the auxiliary verb to describe the noun, and will be created into an attribute in the Class diagram, etc. 2) Relationships within the model, for example, the relationship between "Customer", an Actor, related to "Create new borrow order", a use case in the use case diagram. "Create new borrow order" will be specified in the use case description under the "Use case name" component, and "Customer" will be specified in the "Actor" component, or for the "Customer" class, which has an association relation with the "borrow order" class, "Customer" will be specified in the CRC Card under the "Class name" component, and association relation with the "borrow order" class will be specified under the Relation component etc. 3) Relationships between models, for example, in the use case diagram with an actor named "Customer", the class diagram must specify the "Customer" class as well, etc. The relationships of natural language sentences and models are displayed in a diagram as shown in Fig. 2, and the relationships between models, as well as the relationships within models, are displayed in a diagram as shown in Fig. 3.

\section{E. Create Rules According to the Context of Properties from the Components of Each Requirement}


After studying the components of each form of requirement, and specified the context of good properties according to [1], the results from the study will be used to create rules for verifying the requirements according to the context of the properties of the research. Table VI displays a number of rules created from good properties used for verification of requirements in the natural language sentence and UML model formats, including the number of rules obtained from the relationships of each requirement $(\mathrm{FNL}=$ Functional natural language, $\mathrm{SNL}=$ Structural natural language, $\mathrm{UC}=$ Use case diagram, UCD = Use case description, CD - Class diagram, CRC - CRC Card).

TABLE VI: NuMBER OF RULES CREATED FROM GOOD PROPERTIES USED IN VERIFICATION OF REQUIREMENTS IN THE NATURAL LANGUAGE SENTENCE AND MODEL FORMS

\begin{tabular}{|c|c|c|c|c|c|c|c|c|c|c|c|c|c|c|c|c|c|c|}
\hline & \multicolumn{6}{|c|}{ Unambiguous } & \multicolumn{6}{|c|}{ Consistent } & \multicolumn{6}{|c|}{ Traceable } \\
\hline & FNL & SNL & $\mathrm{UC}$ & UCD & $\mathrm{CD}$ & $\begin{array}{c}\text { CR } \\
\text { C }\end{array}$ & FNL & SNL & $\mathrm{UC}$ & UCD & $\mathrm{CD}$ & $\begin{array}{c}\mathrm{CR} \\
\mathrm{C}\end{array}$ & FNL & SNL & $\mathrm{UC}$ & UCD & $\mathrm{CD}$ & $\begin{array}{c}\text { CR } \\
\mathrm{C}\end{array}$ \\
\hline FNL & 1 & & & & & & & & 6 & & & & & & 2 & & & \\
\hline SNL & & 1 & & & & & & & & & 6 & & & & & & 2 & \\
\hline $\mathrm{UC}$ & & & & & & & & & & 3 & & & & & & 2 & & \\
\hline UCD & & & & 1 & & & & & & & & & & & & & & \\
\hline $\mathrm{CD}$ & & & & & & & & & 1 & & & 6 & & & 2 & & & 2 \\
\hline CRC & & & & & & 1 & & & & & & & & & & & & \\
\hline
\end{tabular}

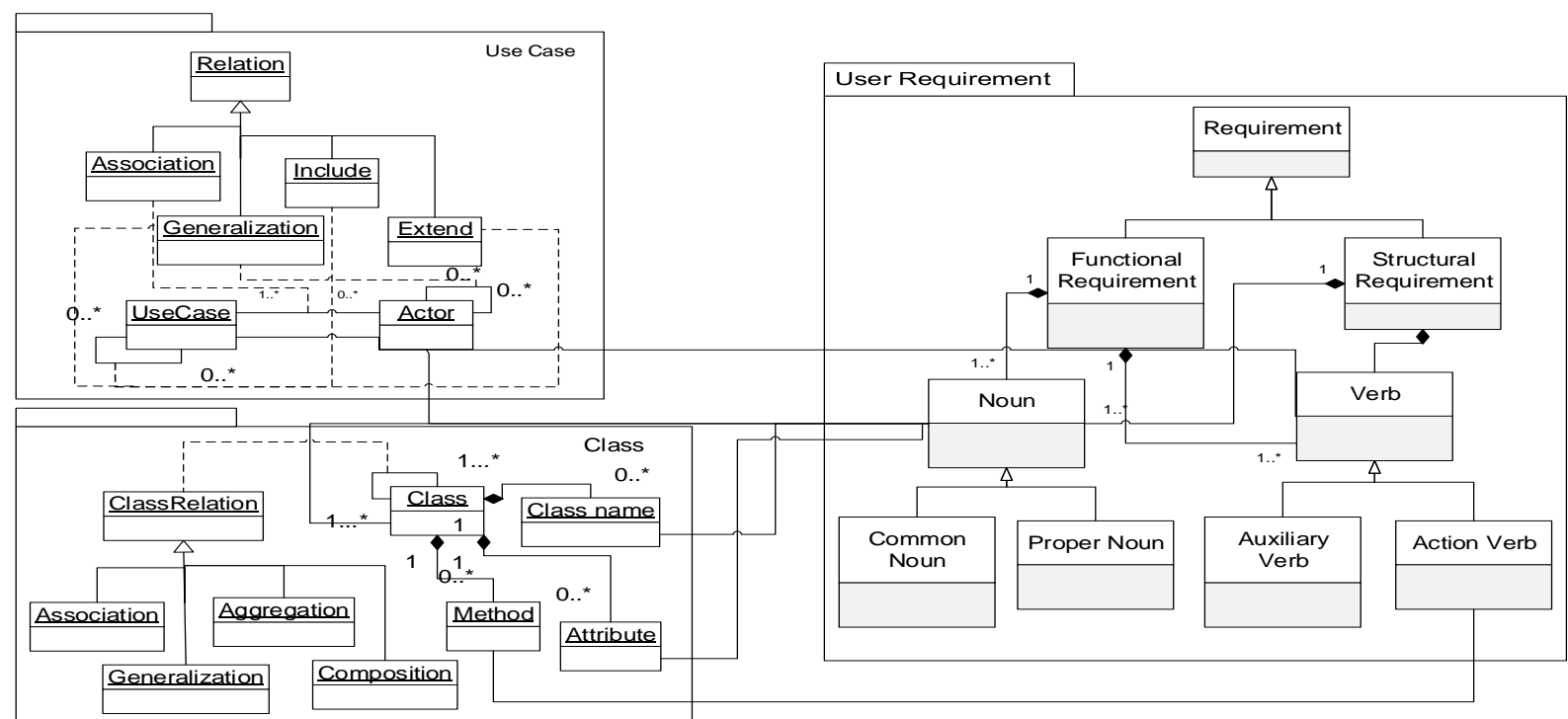

Fig. 2. Relationship diagrams for the components of a natural language sentence and the UML model.

In the next section, examples of applying the presented rules for verifying requirements in the natural language sentence format and requirements in the model format, according to the defined good properties, will be described.

\section{1) Unambiguity}

The unambiguity property has 4 rules created, with an example of a rule and its application as follows:

Example rule: The list of requirements has the unambiguity property only when in the natural language sentence describing the details of the use case under the description component, there are no immeasurable qualifiers.

Example of application: The natural language sentence that appears in the description component of the Use case description as: "Customer can borrow many loan items such as CD and books." This sentence has an immeasurable qualifier, "many", that is, it cannot be clearly specified how many loan items the user can loan. Therefore, when the rule is used for verification, it will find that the use case description is ambiguous. The modification process to clarify the requirements must be performed.

\section{2) Consistency}

The consistency property has 22 rules created, with examples of rules and their applications as follows:

1) Contradictions of the relationships of the tokens between the lists of requirements in the natural language sentence format and the components within the model were analyzed to construct inconsistency checking rules.

Example rule: The list of requirements has the consistency property only when, in the functional natural language sentence, there is a subject with a predicate relationship with the object, appearing as an actor with an association relationship with the use case.

2) Contradictions of calls in the service provided by the system, and the specification of multiplicity values

Example rule: The list of requirements has the consistency property only when, in the structural natural language sentence, there is an enumerated association relationship used as a system specification, appearing as the multicity value of the association relationship in the class diagram.

3) Contradictions of words indicating tokens in the natural language sentence and the model were analyzed to construct inconsistency verification.

Example rule: The list of requirements has the consistency property only when, in the functional natural language sentence, there is a noun acting as the subject of the sentence, appearing as an actor component in the use case diagram.

Example of application: From the functional natural language sentences and the use case model in Fig. 4:

"Customer should be able to borrow loan item. Customer 
should be able to reserve loan item. Customer should be able item." to renew loan item. Librarian should be able to issue loan

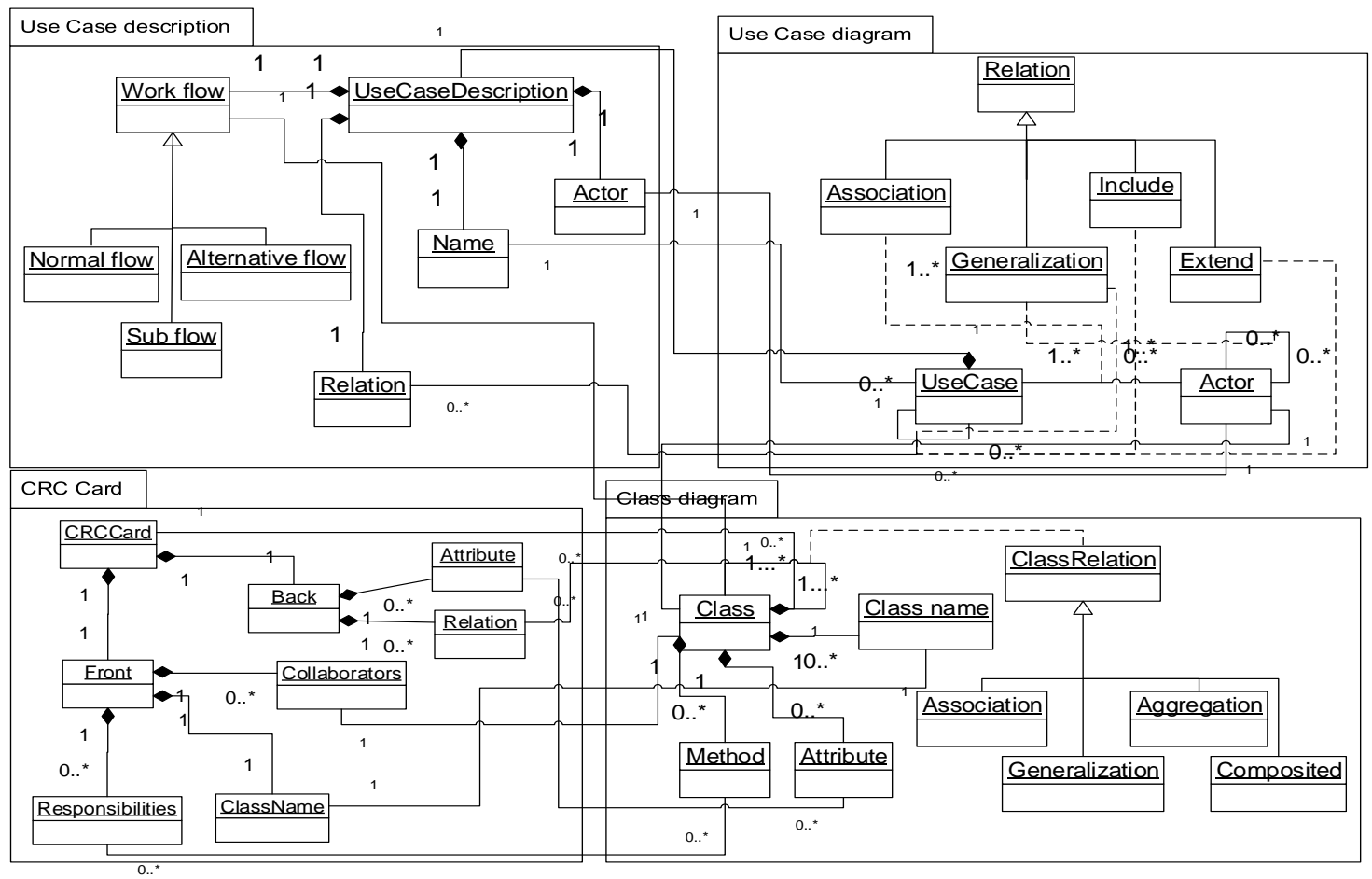

Fig. 3. Conceptual diagram illustrated the relationships of software model components and description.

TABLE VII: EXAMPLE USE CASE DESCRIPTION

\begin{tabular}{|c|c|}
\hline Type & Description \\
\hline Name & Borrow loan item \\
\hline Actor & Librarian, Customer \\
\hline Description & $\begin{array}{l}\text { Customer can borrow many loan items such as CD } \\
\text { and books. }\end{array}$ \\
\hline Relation & - \\
\hline Normal flow & $\begin{array}{l}\text { 1. User logins to the system through login } \\
\text { use case. } \\
\text { 2. } \\
\text { 3. } \\
\text { 4. User searches books from search list. } \\
\text { User summits borrow items. }\end{array}$ \\
\hline Sub flow & $\begin{array}{l}\text { 4s. If user summits loan item, the system alerts } \\
\text { window "Success". }\end{array}$ \\
\hline $\begin{array}{l}\text { Alternative } \\
\text { flow }\end{array}$ & $\begin{array}{l}\text { 4a. If number of loan item }>8 \text { The system will alerts } \\
\text { window "customer has borrowed loan item more than } \\
8 \text { items". }\end{array}$ \\
\hline
\end{tabular}

In the use case model, there is a relationship between the actor named "Customer" and the use case named "Issues loan item", while in the natural language sentences, the subject named "Customer" does not appear with the relationship of the verb "Issues loan item". Therefore, when the rule is used for verification, it finds that the functional natural language sentences and the use case model lack the consistency property.

\section{3) Traceability}

The traceability property has 10 rules created, with examples of rules and their applications as follows:

\section{a) back tracing}

Example rule: The requirements list has the back tracing property only when in the class diagram, class components can be traced to the nouns in the structural natural language sentence.

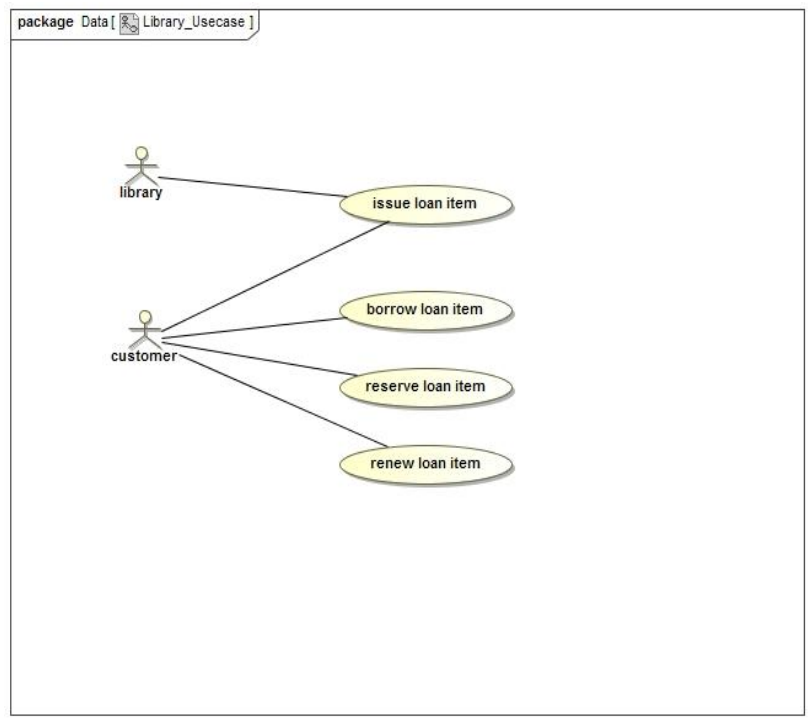

Fig. 4. Use case model.

\section{b) forward tracing}

Example rule: The requirements list has the forward tracing property only when in the functional natural language sentence, there appear nouns acting as classes that can be traced to the class component in the class diagram.

Example of application: From the structural natural language sentences and the class model in Fig. :

"A Librarian issues loan item to each customer. There are exactly two types of loan items, language tapes and books. A language tape has a title language and a label. A book has a title and author(s). It is possibility that each customer may borrow at most 8 items. It is possible that each item can be borrowed, reserved or renewed by customer." 


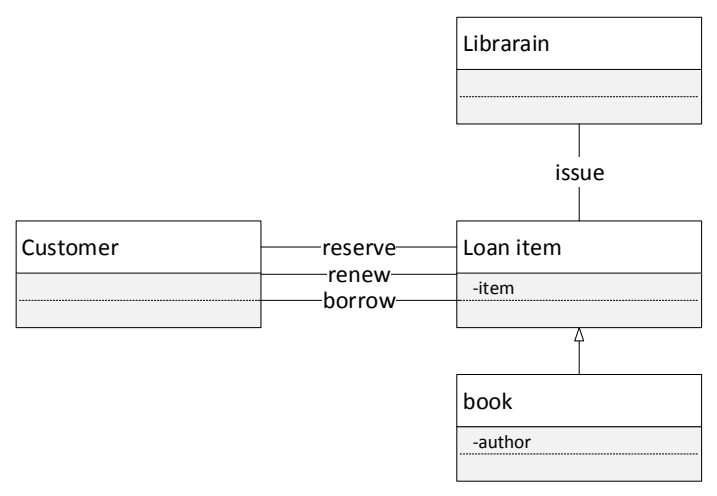

Fig. 5. Structural model.

In the natural language sentences, there appears the noun "language tapes", indicating a class in the class diagram, but the class model does not show a class named "language tapes". Therefore, when the rule is used for verification, it will find that the structural natural language and the class model lack the traceability property.

\section{F. Evaluating the Proposed Rules Used for Verification}

The rules used for verification of requirements in the natural language sentence format, and requirements in the UML model format, created from the relationships in each component of each requirement, are evaluated based on two criteria: accuracy and completeness, with the details of the verification as follows:

Accuracy will be evaluated by applying the rules for verification to look for errors. The rules must be able to identify errors and the method for property verification must follow the context of the research based on each component relationship among models. The application of each property shows the correctness of the rules used for verification, and the verified rules can identify errors in each property.

Completeness will be evaluated by checking whether the components of each requirement can be used to create complete relationships, for creating rules for verification. The relationship and the conceptual diagrams in Fig. 2 and Fig. 3 show the relationship of each component of each requirement. The diagrams show that each component of the requirements in the natural language sentence format, and the components of the model have been studied and have had relationships created, and each component of the requirements in the model format have been studied and have had complete relationships between models and within the model created.

\section{SUMMARY}

This research has presented rules used for verification of requirements in the natural language sentence format and requirements in the UML model format. The rules were created from the study and specification of the context of properties used for verification, the study of natural language sentence structure, the structure of the components in each model, as well as the model description, then taking each component and creating relationships for each requirement based on the studied components, as well as creating rules according to the context of properties from the components of each requirement, according to the 3 relationships of various components of the natural language sentences and the models, namely: 1) The relationship between the natural language sentences and the model 2) The relationships within the model 3) The relationships between the models. From these relationships, 36 rules for verification were created, classified as 4 rules for the unambiguity property, 22 rules for the consistency property, and 10 rules for the tracing property. Using the rules to verify the requirements in various formats can identify which good properties according to the context of the research are missing from each requirement, allowing software developers to update their requirements lists to have good properties according to the standard, which will result in the list of requirements having quality and allowing the software developers to develop software fulfilling user requirements.

In order for the rules proposed in this research to be applied effectively, an approach for future research is to create an automatic tool for using the created rules for verification and identify which properties are missing from various requirements.

\section{REFERENCES}

[1] IEEE Recommended Practice for Software Requirements Specifications, IEEE Std. 830-1998, pp. 1-40, 1998.

[2] L. Xiaoshan, L. Zhiming, and H. Jifeng, "Consistency checking of UML requirements," in Proc. ICECCS 2005, 2005, pp. 411-420.

[3] F. Mokhati, P. Gagnon, and M. Badri, "Verifying UML diagrams with model checking: A rewriting logic based approach," in Proc. QSIC '07, 2007, pp. 356-362.

[4] T. Li et al., "UCVSC: A formal approach to UML class diagram online verification based on situation calculus," in Proc. ICCIT '09, 2009, pp. $375-380$.

[5] A. Umber and I. S. Bajwa, "Minimizing ambiguity in natural language software requirements specification," in Proc. Sixth International Conference on Digital Information Management, 2011, pp. 102-107.

[6] H. Afreen, I. S. Bajwa, and B. Bordbar, "SBVR2UML: A challenging transformation," Frontiers of Information Technology (FIT), 2011, pp. 33-38.

[7] D. P. Tegarden, A. Dennis, and B. H. Wixom, Systems Analysis and Design with UML, Wiley, 2013

[8] I. F. Alexander and L. Beus-Dukic, Discovering Requirements: How to Specify Products and Services, Wiley, 2009.

[9] OMG. (2008). Semantics of Business Vocabulary and Rules. (SBVR) Standard v.1.0. Object Management Group. [Online]. Available: http://www.omg.org/spec/SBVR/1.0/

[10] OMG. (2008). Semantics of Business Vocabulary and Rules. (SBVR) Standard v.1.2. Object Management Group. [Online]. Available: http://www.omg.org/spec/SBVR/1.2/

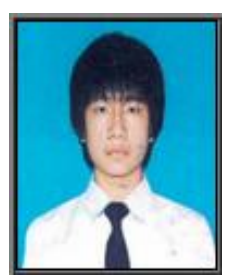

Nattapon Phanthanithilerd is a MSc student in software engineering at Chulalongkorn University. His research interests include software requirements engineering, software process improvement and artificial intelligence. He received a BSc in computer science from Thammasat University.

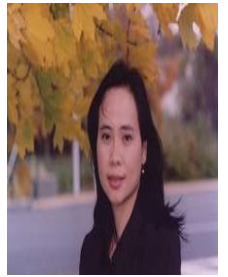

Nakornthip Prompoon is an assistant professor of computer engineering at Chulalongkorn University. Her research interests include software requirements engineering, software process engineering and improving and information storage and retrieval. Prompoon received a MSc in computer science from George Washington University and MBA from Chulalongkorn University. 